\title{
Computer Aided Design of Wing with Given Characteristics
}

\author{
V.M. Sineglazov \\ Aviation Computer-Integrated Complexes Department \\ National Aviation University \\ Kyiv, Ukraine \\ svm@nau.edu.ua
}

\author{
A.A. Ziganshin \\ Aviation Computer-Integrated Complexes Department \\ National Aviation University \\ Kyiv, Ukraine \\ anwarzihan@gmail.com
}

\begin{abstract}
The range of geometric parameters describing the airfoil form was determined. The computer aided design method to optimize these geometric parameters on the base of genetic algorithm was created.
\end{abstract}

Keywords—aerodynamics;wing, airfoil; Darrieus rotor; genetic algorithm; unmanned air vehicle

\section{INTRODUCTION}

At present time great attention is paid to the computer aided design of wings to provide their optimal parameters. Similar tasks emerge at designing air vehicles, especially unmanned air vehicles and wind turbine rotors with vertical axis, particularly Darrieus rotors for increasing their efficiency. The airflow of such wings takes place at low subsonic speeds with Mach numbers beneath 0.3 so that corresponds to critical Reynolds numbers when laminarturbulent transition occurs. Since these wings have great aspect ratios task is converging to finding the optimal form and optimal geometric parameters of the airfoil.

When constructing the optimal airfoils they usually try to achieve the greatest lifting force, the least drag force or the greatest lift-drag ratio at given angle of attack. As it is known it may be attained only in the absence of airflow stall. Because of it another more task arises for extending the range of angles of attack without stall.

\section{TASK STATEMENT}

As parameters defining airfoil are chosen (Fig. 1).

- Chord $b$ (maximal distance between the airfoil outermost points).

- Thickness $c$ (maximal thickness crosswise the chord).

- Camber $f$ (maximal distance between the middle line and the chord).

- Distance $L_{1}$ indicating the position of the maximal thickness point alongside the chord.

- Rounding diameter $D_{1}$ of the leading edge.

- Angles $B_{1}$ and $B_{2}$.
- Angles $A_{1}$ and $A_{2}$ between the tangents to the airfoil edge and the chord.

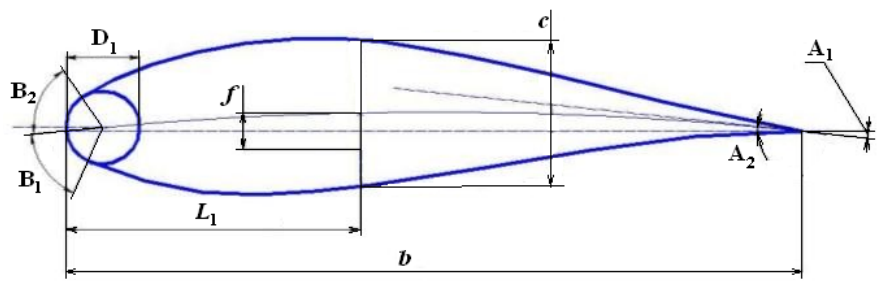

Fig. 1. Parametrical geometry of airfoil.

Geometric parameters of the airfoil affect on its aerodynamic characteristics.

- Increase of the thickness augments the drag. On the other hand, thickness increase causes the stall at the bigger angles of attack, than the thin airfoils have. Increasing of the thickness from the small values to 12 - $15 \%$ enhances the maximum value of lift force coefficient $C_{y}$ Further increasing declines it. Drag force coefficient $C_{x}$ harshly rises after $20 \%$.

- Rounding radius of the airfoil leading edge depending on the thickness affects firstly affects airfoil behavior at the critical angles $\alpha_{c r}$ of attack. It also indirectly influences on drag.

- Camber profile leads to asymmetry of the aerodynamics. Increasing of camber causes growth of the $C_{y}$ at the relatively small values of $R e$. It is necessary to decrease the camber for saving the acceptable values of the drag with growth of $R e$.

The statement of the task is to find airfoils providing the maximum of two criteria:

$$
\begin{aligned}
& \text { Find } \max _{\mathbf{x}}\left\{C_{y}(\mathbf{X}), \alpha_{c r}(\mathbf{X})\right\}=\max _{\mathbf{X}} \mathbf{F}(\mathbf{X}), \\
& \mathbf{X}=\left\{L_{1}, f, c, D_{1}, B_{1}, B_{2}, b, A_{1}, A_{2}\right\}
\end{aligned}
$$

with the restrictions: 


$$
\left.\begin{array}{c}
L_{1 \text { min }}<L_{1}<L_{1 \max }, \\
f_{\text {min }}<f<f_{\max }, \\
c_{\text {min }}<c<c_{\max }, \\
D_{1 \text { min }}<D_{1}<D_{1 \max }, \\
B_{1 \text { min }}<B_{1}<B_{1 \max }, \\
B_{2 \text { min }}<B_{2}<B_{2 \max }, \\
b=b_{0}, \\
A_{1}=A_{2}=A_{0} .
\end{array}\right\}
$$

\section{REVIEW OF EXISTING SOLUTIONS}

The complexity of the task is connected firstly with the fact that the stream parameters are defined after solution for the systems of the non-linear equations with the particular derivatives. As a rule the demands to the subsonic airflows do not allow to use simplified task settings, which got the wide spread in the solution of the supersonic aerodynamics optimization tasks, obtained in the context of the linear theory, Newton, Newton-Buseman streams [1]. That is why it is necessary to use either Euler equations (including the total potential equations) or Navier-Stocks ones. In the case of using Euler equations it is essential to engage the boundary layer equations for the taking into account the viscous effects.

The construction of the optimal solution (the plane flow for the airfoil) requires complex application of the numerical and analytical methods of the mechanics and applied mathematics. These methods can be subdivided into variation, inverse and direct ones.

The range of demands formulated in the task statement restricts application of classic variation methods to construct the optimal solving. Serious difficulties appear taking into account circulation, restrictions having local nature, etc. even using non-viscous gas model. Actually only the work [2] may be noted where it was built a class of symmetrical airfoils with fixed leading and trailing edges and those were optimal by critical Mach number. The method is based on the solution of potential airflow and variation principle generalizing Ryabushinski variaton one [3] for the airflow without circulation over solid bodies.

The methods solving inverse task have long standing history and continue developing now. Irrefragable review in this field for the last twenty years was given in [4]. The essence of the methods and results of solving the inverse boundary problem of plain airflow tasks is to determine the form of the airfoil from the speed field or the pressure one given alongside the airfoil contour. Mathematical models of the ideal liquid flow, boundary layer and Chaplygin gas are used. Along with the solutions of the basic inverse boundary problem variation ones for subsonic airflow, in particular the problem of maximizing the lifting force or fineness, minimizing the airfoil drag using Chaplygin gas and boundary layer models were investigated. Analytical works conducting investigations on the qualitative structure of flow as [5] are rare and investigators must rely on intuition and experience.
As an alternative to inverse design there are the direct methods using the computational methods of optimization based on sequential and repeated solving the airflow tasks with consequent airfoil modification for achieve the characteristics near to demanded ones. These methods do not require a priori information about the solution (pressure and velocity distribution alongside the airfoil contour) and have the most common nature allowing to set and to solve good stated tasks. An additional advantage of using the direct optimization methods is that they may be easily included into multiparameter optimization tasks with potential possibility of taking into account the heterogeneous factors [6]. Direct methods are preferable when angles of attack and flow speed are ever varying.

The general obstacle to using such an optimization procedure is their high cost concerned with the calculation and the analysis of derivatives (or gradients) behavior with the parameters of design. This reason is directly connected with choice of both flow models and calculation method of solving the corresponding equations.

Naturally it is preferable using the Navier-Stokes equations to obtain the most adequate solution of the flow over the airflow.

Several problems encounter when solving the NavierStokes equations: the laminar turbulent transition, the usage of the various turbulence models, the airflow over the trailing edge (for sharp, blunt and rounded edges, bound and shed turbulent layer) [7] and airflow modeling at low Reynolds numbers. It demands great computer resources. Furthemore the researches on the zonal approach do not stop. Here are used various models combining both Euler and Navier-Stokes ones [8], both Euler and boundary layer [9], [10]. The aim of these investigations is a diminution in calculation time with keeping the acceptable accordance of viscous flow equations solution with the experiment.

\section{SOLUTION METHOD}

The genetic algorithm (GA) is the effective way to solve the multicriterion optimization problems. It is a vital member within the family of biologically inspired algorithms. As a matter of fact it is a reproduction model of biological organisms intended to find the global extremum of the multicriterion problems. Mechanisms of the natural selection and the genetics lie on the base of GAs realizing "survival of the strongest" among the structures being considered in their evolutionary process. The general difference of the optimization process with GA is that they work not with the parameters (synaptic weights) but with the encoded set of the parameters. Thus search is carrying out from the population of the initial points. For the quality estimation it is used not the objective increment but its direct instantaneous value applying definite probabilistic rules.

Genetic algorithms were introduced by J. Holland [11] and from the formal point of view were the sequence of operations modeling evolutionary processes on the base of analogues of genetic inheritance mechanism and of natural but sometimes artificial selection. On general view GA may be presented by the chart (Fig. 2). 


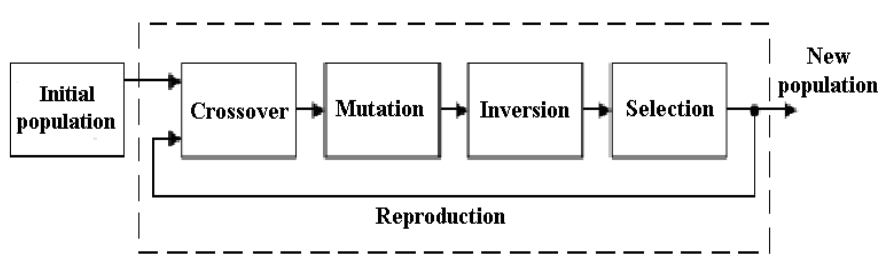

Fig. 2. Chart of genetic algorithm.

The biological terminology is used to describe GA. The key notion is chromosome (string) corresponding the vector (sequence, chain) generated by zeros and ones every position (bit) of which is called gene. The vector of customizable synaptic weights is presented by chromosomes $x_{j}(k)=\left\{x_{j 1}(k), x_{j 2}(k), \ldots, x_{j n}(k)\right\}^{\mathrm{T}}$ being encoded either in binary format or floating point one. If $N$ bits are used to encode customized parameter $x_{j i}(k)$ the chromosome corresponding to the vector of synaptic weights $x_{j}(k)$ has $n N$ genes.

It is necessary at first to generate (usually randomly) an initial chromosome population $X_{j}(0)=\left\{{ }_{1} x_{j}^{\mathrm{T}}(0), \ldots,{ }_{p} x_{j}^{\mathrm{T}}(0)\right.$, $\left.\ldots,{ }_{Q} x_{j}^{\mathrm{T}}(0)\right\}$ (here ${ }_{p} x_{j}^{\mathrm{T}}(0)=\left\{x_{j 1}(0),{ }_{p} x_{j 2}(0), \ldots,{ }_{p} x_{j n}(0)\right\}$ is the $p^{\text {th }}$ chromosome population). Its dimension $Q$ is often supposed to be a constant one. The fitness may be estimated for every generated chromosome and it is the objective value $E_{j}\left({ }_{p} x_{j}(0)\right)$ for the $p^{\text {th }}$ vector of synaptic weights. It is evident that the $p^{\text {th }}$ chromosome has the better chance of "surviving" in evolving population if it has the more value of $E_{j}\left({ }_{p} x_{j}(0)\right)$.

Then the population reproduction process begins being generated by the genetic operators of the crossover, mutation and inversion and the selection operation.

The most important operator is the crossover generating the descendant chromosomes by the genetic exchange between the parental chromosomes.

A great number of crossover variants exists the simplest of which is the single point one where the randomly chosen parents are cut over in the randomly chosen point after what the chromosomes exchange their fragments. Generally the single point crossover is ineffective whereas it leads to the change of only one synaptic weight ${ }_{p} x_{j i}(k)$ in the string ${ }_{p} x_{j}(k)$. It is necessary to use the $n$-point crossover in which more than two parental chromosomes can take part for changing all the weights.

The mutation is connected with the randomly change of one or more genes of chromosome. Here the randomly chosen bit changes its state to opposite one. Mutation operator does not allow the learning process "to stick" in the local extremums of the objective function.
The inversion operator changes the order of genes in the chromosome by the way of cyclic permutation. Though the inversion is not often used in optimization tasks but it allows to change all the synaptic weights of the string ${ }_{p} x_{j}(k)$.

An extended chromosome population is generated as result of crossovers, mutations and inversions. It consists of both the initial ensemble of parental chromosomes and descendant ones. Every string of the extended population is estimated for its fitness by criterion $E_{j}\left(x_{p} x_{j}(0)\right), p=1,2, \ldots, Q, \ldots$ after that a new population is generated $X_{j}(1)$ containing $Q(1)$ chromosomes with the greatest values of $E_{j}\left({ }_{p} x_{j}\right)$. The essence of selection operation is in it.

Then the reproduction process repeats cyclically on the every $k^{\text {th }}$ iteration. Thus GA accumulates the successful solutions "drawing up" population to the global extremum of the objective function (Pareto set).

\section{CONCLUSIONS}

The range of geometric parameters describing the airfoil form was determined.

The computer aided design method to optimize these geometric parameters on the base of genetic algorithm was created.

\section{REFERENCES}

[1] A. Fox and M. Pratt, Numerical geometry. Application in design and production. Moscow, Mir, 1982, 304 p. (In Russian).

[2] N.N. Vabishevich, "Adaptive grids of compound type in mathematical physics tasks," Journal of applied mathematics and mathematical physics. 1989, vol. 20, no. 6, pp. 902-914. (In Russian).

[3] V.A.Vasilenko, Spline functions: Theory, algorithms, programs. Novosibirsk, Nauka, 1983, 215 p. (In Russian).

[4] S. A. Ivanenko, "Adaptive grids and grids on surfaces," Journal of applied mathematics and mathematical physics. 1993, vol. 33, no. 9, pp. 1333-1351. (In Russian)

[5] J.O.Hager, S.Eyi and K.D.Lee, Multipoint design of transonic airfoils using optimization, AIAA Pap, 1992, no. 4225, pp. 1-9.

[6] S.M.Aulchenko and A.F. Latypov, "Modelling airfoils in subsonic airflow by methods of numerical optimization," Liquid and gas mechanics, 1997, no. 2, pp.174-182. (In Russian).

[7] J.F. Thompson, "A survey of dynamically adaptive grids in the numerical solution of partial differential equations," Appl. Numer. Math. 1985, vol. 1, pp. 3-27.

[8] W.C. Thacher, "A brief review of techniques for generations irregular computational grids," Internal J. Numer. Meth. Engng. 1980, vol. 15, no. 9, pp. 1335-1342.

[9] K.D. Lee and Y.M. Tsuei, "A hybrid adaptive gridding procedure for recirculating fluid flow problems," J. Comput. Phys. 1993, vol. 108, no. 1, pp. 122-141.

[10] K.D. Lee and P.H.Liu, "A design optimization method using the Euler equations," Numer. Meth. Laminar and Turbulent Flow: Proc. 6 th Int. Conf, 1989, vol. 6, Pt 1, pp.773-872.

[11] J.H. Holland, Adaptation in Natural and Artificial Systems. An Introductory Analysis with Application to Biology, Control and Artificial Intelligence. London: Bradford Book Edition, 1994, 211 p. 